Article

\title{
On the product rule for the hyperbolic scator algebra
}

\author{
Jan L. Cieśliński (1), Artur Kobus
}

Uniwersytet w Białymstoku, Wydział Fizyki, ul. Ciołkowskiego 1L, 15-245 Białystok, Poland; a.kobus@uwb.edu.pl

* Correspondence: j.cieslinski@uwb.edu.pl

\begin{abstract}
Scator set, introduced by Fernández-Guasti and Zaldívar, is endowed with a very peculiar non-distributive product. In this paper we consider the scator space of dimension $1+2$ and the so called fundamental embedding which maps the subset of scators with non-zero scalar component into 4-dimensional space endowed with a natural distributive product. The original definition of the scator product is induced in a straightforward way. Moreove, we propose an extension of the scator product on the whole scator space, including scators with vanishing scalar component.
\end{abstract}

Keywords: scators; non-distributive algebras; Lorentz velocity addition formula; fundamental embedding

MSC: 30G35; 20M14

\section{Introduction}

The scator algebra was introduced by Fernández-Guasti and Zaldívar in a series of papers, starting from [1,2]. In this paper we confine ourselves to scators in the hyperbolic space of dimension $1+2$, i.e., to the objects of the following form:

$$
\stackrel{o}{a}=\left(a_{0} ; a_{1}, a_{2}\right)=a_{0}+a_{1} \stackrel{o}{\boldsymbol{e}_{1}}+a_{2} \stackrel{o}{\boldsymbol{e}_{2}}
$$

where $a_{0}, a_{1}$ and $a_{2}$ are real numbers and $\stackrel{o}{\boldsymbol{e}}_{1}$ and $\stackrel{o}{\boldsymbol{e}}_{2}$ are basis unit vectors. In the hyperbolic case $\left(\stackrel{o}{\boldsymbol{e}}_{1}\right)^{2}=$ $\left(\boldsymbol{e}_{2}\right)^{2}=1$. However, we point out that the notion of the product of scators is rather unusual and will be defined and clarified below. The coefficient $a_{0}$ is referred to as the scalar component and $a_{1}, a_{2}$ are called director components. The elliptic case (when squares of basis vectors are negative) can be considered as an example of hypercomplex numbers [2,3], while the hyperbolic case has potential physical applications related to deformations and generalizations of the special theory of relativity [4,5], see also [6-8].

Definition 1. Product of two scators, $\stackrel{o}{a}=\left(a_{0} ; a_{1}, a_{2}\right)$ and $\stackrel{o}{b}=\left(b_{0} ; b_{1}, b_{2}\right)$, denoted by $\stackrel{o^{o}}{b} \equiv\left(u_{0} ; u_{1}, u_{2}\right)$, is defined as follows [1].

- For $a_{0} \neq 0$ and $b_{0} \neq 0$,

$$
\begin{aligned}
& u_{0}=a_{0} b_{0}+a_{1} b_{1}+a_{2} b_{2}+\frac{a_{1} a_{2} b_{1} b_{2}}{a_{0} b_{0}} \equiv a_{0} b_{0}\left(1+\frac{a_{1} b_{1}}{a_{0} b_{0}}\right)\left(1+\frac{a_{2} b_{2}}{a_{0} b_{0}}\right), \\
& u_{1}=a_{0} b_{1}+a_{1} b_{0}+\frac{a_{1} a_{2} b_{2}}{a_{0}}+\frac{a_{2} b_{1} b_{2}}{b_{0}} \equiv a_{0} b_{0}\left(\frac{a_{1}}{a_{0}}+\frac{b_{1}}{b_{0}}\right)\left(1+\frac{a_{2} b_{2}}{a_{0} b_{0}}\right), \\
& u_{2}=a_{0} b_{2}+a_{2} b_{0}+\frac{a_{1} a_{2} b_{1}}{a_{0}}+\frac{a_{1} b_{1} b_{2}}{b_{0}} \equiv a_{0} b_{0}\left(1+\frac{a_{1} b_{1}}{a_{0} b_{0}}\right)\left(\frac{a_{2}}{a_{0}}+\frac{b_{2}}{b_{0}}\right) .
\end{aligned}
$$


- For $a_{0}=a_{2}=0$ and $a_{1}=1$,

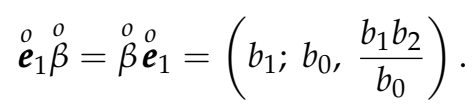

- For $a_{0}=a_{1}=0$ and $a_{2}=1$,

$$
\stackrel{o}{\boldsymbol{e}_{2}} \beta \stackrel{o}{\beta}=\stackrel{o}{\beta} \stackrel{o}{\boldsymbol{e}_{2}}=\left(b_{2} ; \frac{b_{1} b_{2}}{b_{0}}, b_{1}\right) \text {. }
$$

- In any case

$$
(\lambda \stackrel{o}{a}) \stackrel{o}{b}=\stackrel{o}{a}(\lambda \stackrel{o}{b})=\lambda(\stackrel{o}{a} a) .
$$

In particular, the hyperbolic basis satisfies

$$
\stackrel{o}{\boldsymbol{e}_{1} \stackrel{o}{\boldsymbol{e}}_{1}}=\stackrel{o}{\boldsymbol{e}_{2} \stackrel{o}{\boldsymbol{e}}_{2}}=1, \quad \stackrel{o}{\boldsymbol{e}_{1} \boldsymbol{e}_{2}}=\stackrel{o}{\boldsymbol{e}_{2} \boldsymbol{e}_{1}}=0,
$$

anticipating commutativity and non-associativity of the scator set under multiplication. Generalization to higher dimensions is obvious, see [3,9].

Remark 2. Using the notation

$$
\begin{aligned}
& \left(a_{0} ; a_{1}, a_{2}\right)=a_{0}\left(1 ; \beta_{a 1}, \beta_{a 2}\right), \\
& \left(b_{0} ; b_{1}, b_{2}\right)=b_{0}\left(1 ; \beta_{b 1}, \beta_{b 2}\right),
\end{aligned}
$$

(compare [10]), we rewrite the generic case (i.e., Eqs. (2)) as:

$$
\begin{aligned}
& u_{0}=a_{0} b_{0}\left(1+\beta_{a 1} \beta_{b 1}\right)\left(1+\beta_{a 2} \beta_{b 2}\right), \\
& u_{1}=a_{0} b_{0}\left(\beta_{a 1}+\beta_{b 1}\right)\left(1+\beta_{a 2} \beta_{b 2}\right), \\
& u_{2}=a_{0} b_{0}\left(1+\beta_{a 1} \beta_{b 1}\right)\left(\beta_{a 2}+\beta_{b 2}\right),
\end{aligned}
$$

In other words, for $a_{0} \neq 0$ and $b_{0} \neq 0$ we have

$$
\stackrel{o}{a} b=a_{0} b_{0}\left(1+\beta_{a 1} \beta_{b 1}\right)\left(1+\beta_{a 2} \beta_{b 2}\right)\left(1 ; \frac{\beta_{a 1}+\beta_{b 1}}{1+\beta_{a 1} \beta_{b 1}}, \frac{\beta_{a 2}+\beta_{b 2}}{1+\beta_{a 2} \beta_{b 2}}\right) .
$$

The components of the formula (9) remind the Lorentz rule for relativistic sum of velocities (in the one-dimensional case), which motivates some physical applications [4,5]. In this paper we present a natural motivation for the complicated formulas of Definition 1. We also we propose a novel extension of Definition 1 on the case $a_{0}=0$ (for any values of $a_{1}$ and $a_{2}$ ). 


\section{Commutativity, non-distributivity and generic associativity of the scator product}

The scator product, as defined above, is manifestly commutative, for any pair of scators. In general it is non-distributive, which is clearly underlined in the first papers on the scator algebra [1,2,9]. In order to explicitly show no-distributivity one can compute [10]:

$$
\Delta(\stackrel{o}{a}, \stackrel{o}{b} ; \stackrel{o}{c}):=(\stackrel{o}{a}+\stackrel{o}{b})_{c}^{o}-\stackrel{o o}{a c}-\stackrel{o}{b c} c=\frac{\left(b_{0} a_{1}-a_{0} b_{1}\right)\left(a_{0} b_{2}-b_{0} a_{2}\right)}{a_{0} b_{0}\left(a_{0}+b_{0}\right)}\left(\frac{c_{1} c_{2}}{c_{0}}, c_{2}, c_{1}\right),
$$

where we denote, as usual, $\stackrel{o}{c}=\left(c_{0} ; c_{1}, c_{2}\right)$. We see, that except some special cases $\left(a_{0} b_{1}=a_{1} b_{0}\right.$ or $\left.a_{0} b_{2}=a_{2} b_{0}\right)$ the scator product is not distributive.

Computing the product of three scators with non-vanishing scalar components, under assumption that the scalar components of $\stackrel{\stackrel{o}{a}^{o}}{a b}, \stackrel{o}{b c}$ and $\stackrel{o o}{a c}$ do not vanish, we obtain

$$
(\stackrel{o}{a b})^{o} c=\stackrel{o}{a}(\stackrel{o}{b c})=\stackrel{o}{b}(\stackrel{o o}{c a})=\left(w_{0} ; w_{1}, w_{2}\right) \equiv w_{0}\left(1 ; \beta_{w 1}, \beta_{w 2}\right),
$$

where

$$
\begin{aligned}
& w_{0}=a_{0} b_{0} c_{0}\left(1+\beta_{a 1} \beta_{b 1}+\beta_{a 1} \beta_{c 1}+\beta_{b 1} \beta_{c 1}\right)\left(1+\beta_{a 2} \beta_{b 2}+\beta_{a 2} \beta_{c 2}+\beta_{b 2} \beta_{c 2}\right), \\
& w_{1}=a_{0} b_{0} c_{0}\left(\beta_{a 1}+\beta_{b 1}+\beta_{c 1}+\beta_{a 1} \beta_{b 1} \beta_{c 1}\right)\left(1+\beta_{a 2} \beta_{b 2}+\beta_{a 2} \beta_{c 2}+\beta_{b 2} \beta_{c 2}\right), \\
& w_{2}=a_{0} b_{0} c_{0}\left(1+\beta_{a 1} \beta_{b 1}+\beta_{a 1} \beta_{c 1}+\beta_{b 1} \beta_{c 1}\right)\left(\beta_{a 2}+\beta_{b 2}+\beta_{c 2}+\beta_{a 2} \beta_{b 2} \beta_{c 2}\right) .
\end{aligned}
$$

Therefore, in the generic case the scator product is associative but in special cases the associativity is broken. The simplest example of the non-associativity is given by triple products of basis vectors.

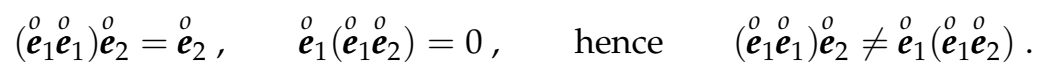

Note that both $\stackrel{o}{e}_{1}$ and $\stackrel{o}{e}_{2}$ are zero divisors.

\section{Fundamental embedding as a natural interpretation of the scator product}

In Ref. [10] we introduced the so called fundamental embedding $F: S^{\prime} \rightarrow A$, where $S^{\prime}$ is subset of the scator space containing scators with non-zero scalar component and the space $A$ is spanned by elements $1, \boldsymbol{e}_{1}, \boldsymbol{e}_{2}$ and $\boldsymbol{e}_{12}$, which satisfy

$$
\boldsymbol{e}_{1} \boldsymbol{e}_{1}=\boldsymbol{e}_{2} \boldsymbol{e}_{2}=1, \quad \boldsymbol{e}_{1} \boldsymbol{e}_{2}=\boldsymbol{e}_{2} \boldsymbol{e}_{1}=\boldsymbol{e}_{12}
$$

We assume that the product in the space $A$ is commuative, associative and distributive over addition. In this paper we will use a slightly more general definition of the fundamental embedding, extending it on a larger subset of the scator space, namely $S^{\prime \prime}$ given as

$$
S^{\prime \prime}=S^{\prime} \cup \operatorname{span}\left\{\boldsymbol{e}_{1}^{o}\right\} \cup \operatorname{span}\left\{\boldsymbol{e}_{2}\right\} .
$$


Definition 3. The fundamental embedding $F: S^{\prime \prime} \rightarrow A$ is defined as:

$$
\begin{aligned}
& F(\stackrel{o}{a})=a_{0}+a_{1} \boldsymbol{e}_{1}+a_{2} \boldsymbol{e}_{2}+\frac{a_{1} a_{2}}{a_{0}} \boldsymbol{e}_{12} \quad\left(a_{0} \neq 0\right), \\
& F\left(a_{1} \boldsymbol{e}_{1}\right)=a_{1} \boldsymbol{e}_{1}, \\
& F\left(a_{2} \boldsymbol{e}_{2}\right)=a_{2} \boldsymbol{e}_{2} .
\end{aligned}
$$

Remark 4. The planes $\left(a_{0} ; 0, a_{2}\right)$ and $\left(a_{0} ; a_{1}, 0\right)$ are invariants of the fundamental embedding:

$$
\begin{aligned}
& F\left(a_{0}+a_{1}{\stackrel{o}{\boldsymbol{e}_{1}}}^{\prime}\right)=a_{0}+a_{1} \boldsymbol{e}_{1}, \\
& F\left(a_{0}+a_{2} \boldsymbol{e}_{2}\right)=a_{0}+a_{2} \boldsymbol{e}_{2} .
\end{aligned}
$$

For $a_{0} \neq 0$ this is a special case of the first formula of (16).

The space $A$ may be understood as a commutative analogue of the geometric algebra or the Clifford algebra [11], where vectors commute only when they are parallel (and orthogonal vectors anti-commute). The image of the fundamental embedding, denoted by $\tilde{S} \equiv F\left(S^{\prime}\right)$, has a natural group structure reminding a commutative analogue of the Clifford (or Lipschitz) group [12]. Indeed, the first equation of (16) can be rewritten as

$$
F(\stackrel{o}{a})=a_{0}\left(1+\frac{a_{1}}{a_{0}} \boldsymbol{e}_{1}\right)\left(1+\frac{a_{2}}{a_{0}} \boldsymbol{e}_{2}\right)
$$

Operations in the space $A$ give a natural interpretation and motivation for the definition (2) of the scator product. The following theorem holds.

Theorem 5. If $a_{0} \neq 0, b_{0} \neq 0, a_{1} b_{1}+a_{0} b_{0} \neq 0$ and $a_{2} b_{2}+a_{0} b_{0} \neq 0$, then there exists $\stackrel{o}{c} \in S^{\prime}$ such that

$$
F(\stackrel{o}{a}) F(\stackrel{o}{b})=F(\stackrel{o}{c}) .
$$

What is more, $\stackrel{o}{c}$ coincides with $\stackrel{o^{o}}{a}$ given by Definition 1, i.e.,

$$
F\left(\stackrel{o^{o}}{a} b\right)=F(\stackrel{o}{a}) F(\stackrel{o}{b}), \quad \stackrel{\stackrel{o}{a}^{o}}{a}=F^{-1}(F(\stackrel{o}{a}) F(\stackrel{o}{b})) .
$$

Proof. First, let us observe that

$$
\left(1+\frac{a_{1}}{a_{0}} \boldsymbol{e}_{1}\right)\left(1+\frac{b_{1}}{b_{0}} \boldsymbol{e}_{1}\right)=1+\frac{a_{1} b_{1}}{a_{0} b_{0}}+\left(\frac{a_{1}}{a_{0}}+\frac{b_{1}}{b_{0}}\right) \boldsymbol{e}_{1}=\left(1+\beta_{a 1} \beta_{b 1}\right)+\left(\beta_{a 1}+\beta_{b 1}\right) \boldsymbol{e}_{1},
$$

where we used the notation (7). Therefore, the product

$$
F(\stackrel{o}{a}) F(\stackrel{o}{b})=a_{0} b_{0}\left(1+\frac{a_{1}}{a_{0}} \boldsymbol{e}_{1}\right)\left(1+\frac{b_{1}}{b_{0}} \boldsymbol{e}_{1}\right)\left(1+\frac{a_{2}}{a_{0}} \boldsymbol{e}_{2}\right)\left(1+\frac{b_{2}}{b_{0}} \boldsymbol{e}_{2}\right)
$$

can be easily represented in the form (18). Indeed,

$$
F(\stackrel{o}{a}) F(\stackrel{o}{b})=a_{0} b_{0}\left(1+\beta_{a 1} \beta_{b 1}+\left(\beta_{a 1}+\beta_{b 1}\right) \boldsymbol{e}_{1}\right)\left(1+\beta_{a 2} \beta_{b 2}+\left(\beta_{a 2}+\beta_{b 2}\right) \boldsymbol{e}_{2}\right)
$$


Denoting $\stackrel{o}{c}:=\left(c_{0} ; c_{1}, c_{2}\right)$, where

$$
\begin{aligned}
& c_{0}=a_{0} b_{0}\left(1+\beta_{a 1} \beta_{b 1}\right)\left(1+\beta_{a 2} \beta_{b 2}\right), \\
& c_{1}=a_{0} b_{0}\left(\beta_{a 1}+\beta_{b 1}\right)\left(1+\beta_{a 2} \beta_{b 2}\right), \\
& c_{2}=a_{0} b_{0}\left(1+\beta_{a 1} \beta_{b 1}\right)\left(\beta_{a 2}+\beta_{b 2}\right),
\end{aligned}
$$

we get Eq. (19). The conditions $a_{1} b_{1}+a_{0} b_{0} \neq 0$ and $a_{2} b_{2}+a_{0} b_{0} \neq 0$ assure that $c_{0} \neq 0$, so $\stackrel{o}{c} \in S^{\prime}$, as needed. One can easily see that the above $\stackrel{o}{c}$ coincides with $\stackrel{o}{u}$ defined by (8), which ends the proof.

Remark 6. Theorem 5 does not extend on all scators from $S^{\prime}$ or $S^{\prime \prime}$. For instance,

$$
\begin{aligned}
& F\left(\stackrel{o}{\boldsymbol{e}_{1}} \stackrel{o}{b}\right)=F\left(\stackrel{o}{\boldsymbol{e}_{1}}\right) F(\stackrel{o}{b}) \quad\left(\text { if } b_{0} \neq 0 \text { and } b_{1} \neq 0\right), \\
& F\left(\stackrel{o}{\boldsymbol{e}_{1}} \stackrel{o}{b}\right) \neq F\left(\stackrel{o}{\boldsymbol{e}_{1}}\right) F(\stackrel{o}{b}) \quad\left(\text { for } \stackrel{o}{b}=b_{0}+b_{2} \boldsymbol{e}_{2}, \quad b_{0} \neq 0\right) \text {. }
\end{aligned}
$$

It is worth noting that conditions $a_{1} b_{1}+a_{0} b_{0} \neq 0$ and $a_{2} b_{2}+a_{0} b_{0} \neq 0$ in Theorem 5 are essential and necessary. If, for instance, $a_{1} b_{1}+a_{0} b_{0}=0$, then $\stackrel{o^{o}}{a}$ and $F\left(\stackrel{o^{o}}{a}\right)$ are proportional to $\boldsymbol{e}_{1}$, while $F(\stackrel{o}{a}) F(\stackrel{o}{b})$ is, in general, a linear combination of $\boldsymbol{e}_{1}$ and $\boldsymbol{e}_{12}$, compare (23).

\section{Extension of the scator product on all scators with vanishing scalar component}

Definition 1 contains only two special cases when one factor can have vanishing scalar component. We may shortly say, that Definition 1 is applicable for all scators belonging to $S^{\prime \prime}$. In this section we propose an extension of this definition on the whole scator space $S$. First, we consider the case when the first factor is of the form

$$
\stackrel{o}{a}=a_{1} \stackrel{o}{\boldsymbol{e}_{1}}+a_{2}{\stackrel{o}{\boldsymbol{e}_{2}},}^{2}
$$

while the seccond factor belongs to $S^{\prime}$ (provided that $b_{0} \neq 0$ ):

$$
\stackrel{o}{b}=b_{0}+b_{1} \stackrel{o}{\boldsymbol{e}_{1}}+b_{2} \stackrel{o}{\boldsymbol{e}_{2}}
$$

We base our treatment on discrete symmetries of the scator set, namely, reflection symmetries. Let us introduce

$$
\stackrel{o}{a_{\varepsilon}}=\varepsilon+a_{1} \stackrel{o}{e}_{1}+a_{2} \stackrel{o}{e}_{2}
$$

where we understand $\varepsilon$ as a very small real positive number. Finally it will approach zero. We will consider products of the $\stackrel{o}{b}$ scator with both $\varepsilon$ and $-\varepsilon$ versions of $\stackrel{o}{a}$. In this way, since product is non-distributive, we have to obtain two disparate results (divergent when $\varepsilon \rightarrow 0$ ) with troublesome terms having opposite signs. Therefore, to find proper (finite) product we define it as

$$
\stackrel{o}{a} b=\lim _{\varepsilon \rightarrow 0} \frac{\stackrel{o}{a_{\varepsilon} b}+\stackrel{o}{b} \stackrel{o}{a}_{-\varepsilon} b}{2}=\left(a_{1} b_{1}+a_{2} b_{2} ; a_{1} b_{0}+\frac{a_{2} b_{1} b_{2}}{b_{0}}, a_{2} b_{0}+\frac{a_{1} b_{1} b_{2}}{b_{0}}\right),
$$

where, after straightforward calculation, limiting procedure acts component-wise and we use scator product defined by (2). 
The last case to consider is the product of two "deficient" scators:

$$
\stackrel{o}{a}=a_{1} \stackrel{o}{\boldsymbol{e}_{1}}+\stackrel{o}{a_{2}}, \quad \stackrel{o}{b}=b_{1} \stackrel{o}{\boldsymbol{e}}_{1}+b_{2} \stackrel{o}{\boldsymbol{e}_{2}} .
$$

Now we have four combinations of divergent terms which also can be cancelled by taking an appropriate sum. In the identical manner as before, we propose in the case (30) the following formula:

$$
\stackrel{o}{a} b=\lim _{\varepsilon, \delta \rightarrow 0} \frac{\stackrel{o}{a_{\varepsilon}} \stackrel{o}{b}_{\delta}+\stackrel{o}{a}_{-\varepsilon} \stackrel{o}{b}_{\delta}+\stackrel{o}{a}_{\varepsilon} \stackrel{o}{b}_{-\delta}+\stackrel{o}{a}_{-\varepsilon} \stackrel{o}{b}_{-\delta}}{4}=a_{1} b_{1}+a_{2} b_{2},
$$

where we use a standard convention $a_{1} b_{1}+a_{2} b_{2} \equiv\left(a_{1} b_{1}+a_{2} b_{2} ; 0,0\right)$.

Finally, we are going to define the fundamental embedding for the scators of the form (26). We use approach similar to what we performed above, i.e.

$$
F(\stackrel{o}{a})=\lim _{\varepsilon \rightarrow 0} \frac{1}{2}\left(F\left(\stackrel{o}{a}_{\varepsilon}\right)+F(\stackrel{o}{a}-\varepsilon)\right)=a_{1} \boldsymbol{e}_{1}+a_{2} \boldsymbol{e}_{2} .
$$

Therefore, the plane $\left(0 ; a_{1}, a_{2}\right)$ is an invariant of the fundamental embedding. Hence, taking into account Remerk 4, all coordinate axes and planes perpendicular to them are invariant with respect to the fundamental embedding $F$.

\section{Conclusions}

We studied multiplication in the scator space of dimension $1+2$. Multiplication of scators is usually restricted to a subset which consists mostly of scators with non-vanishing scalar component. In this paper we proposed and discussed an extension of the scator product on the whole scator space. Theorem 5 gives a motivation and interpretation for the commonly used definition of this product (Definition 1) which otherwise is rather not very obvious. In the generic case the scator product is induced by another product (in another space) which is commutative, associative and distributive over addition.

Author Contributions: conceptualization, J.L.C. and A.K.; methodology, J.L.C. and A.K.; formal analysis, J.L.C.; investigation, J.L.C. and A.K.; writing-original draft preparation, A.K.; writing-review and editing, J.L.C.

Funding: This research received no external funding.

Conflicts of Interest: The author declares no conflict of interest.

\section{References}

1. M.Fernández-Guasti, F.Zaldívar: A Hyperbolic Non-Distributive Algebra in 1+2 dimensions, Adv. Appl. Clifford Algebras 23 (2013) 639-656.

2. M.Fernández-Guasti, F.Zaldívar: An elliptic non distributive algebra, Adv. Appl. Clifford Algebras 23 (4) (2013) 825-835.

3. M.Fernández-Guasti: A Non-distributive Extension of Complex Numbers to Higher Dimensions, Adv. Appl. Clifford Algebras 25 (2015) 829-849.

4. M.Fernández-Guasti: Time and space transformations in a scator deformed Lorentz metric, Eur. Phys. J. Plus (2014), 129: 195.

5. M.Fernández-Guasti, F.Zaldívar: Hyperbolic superluminal scator algebra, Adv. Appl. Clifford Algebras 25 (2015) 321-335. 
6. J. Magueijo, L. Smolin: Generalized Lorentz invariance with an invariant energy scale, Phys. Rev. D 67 (2003) 044017.

7. G. Amelino-Camelia: Doubly-Special Relativity: Facts, Myths and Some Key Open Questions, Symmetry 2 (2010) 230-271.

8. E. Kapuścik: On Fatal Error in Tachyonic Physics, Int. J. Theor. Phys. 54 (2015) 4041-4045.

9. M.Fernández-Guasti, F.Zaldívar: Multiplicative representation of a hyperbolic non distributive algebra, Adv. Appl. Clifford Algebras 24 (3) (2014) 661-674.

10. A. Kobus, J.L. Cieśliński: On the Geometry of the Hyperbolic Scator Space in $1+2$ Dimensions, Adv. Appl. Clifford Algebras 27 (2017) 1369-1386.

11. D. Hestenes, G. Sobczyk: Clifford Algebra to Geometric Calculus: A Unified Language for Mathematics and Physics, D. Reidel Publ. Co., Dordrecht 1984.

12. J.L.Cieśliński: Divisors of zero in the Lipschitz semigroup, Adv. Appl. Clifford Algebras 17 (2) (2007) $153-157$. 